\title{
NEUTRON SCATTERING STUDIES OF MAGNETIC CORRELATIONS IN THE LAYERED CUPRATES
}

\author{
J. M. TRANQUADA and G. SHIRANE \\ Physics Department, Brookhaven National Laboratory, Upton, NY 11973, USA
}

All of the copper oxide superconductors contain $\mathrm{CuO}_{2}$ layers which, when insulating, exhibit strong, twodimensional (2D) antiferromagnetic correlations due to the unusually large superexchange interaction between nearest-neighbor $\mathrm{Cu}^{2+}$ atoms. Magnetic correlations survive, although with a reduced correlation length, even when the layers are doped with holes. A short review of neutron scattering studies of magnetic correlations and excitations in the layered cuprates is presented.

By doping the $\mathrm{CuO}_{2}$ planes with holes (or electrons), it is possible to transform a number of layered copper oxide compounds from antiferromagnetic insulators to metallic superconductors. There is clearly a close connection between riagnetism and superconductivity in the cuprates, and it is of considerable interest to deternine the extent to which superconductivity competes with or depends upon the magnetism. Neutron scattering is a powerful probe which has provided considerable insight into the nature of magnetic correlations in both the metallic and insulating phases of the cuprates. In this paper, we will give a brief review of the picture obtained from neutron scattering studies of $\mathrm{La}_{3-2} \mathrm{Sr}_{2} \mathrm{CuO}_{1+y}$ and $\mathrm{YBa}_{2} \mathrm{Cu}_{3} \mathrm{O}_{0+x}$.

The effect of doping on the magnetic correlations within a $\mathrm{CuO}_{2}$ plane will depend to a large extent on the nature of the antiferromagnetic state which is found in the insulating phase. It is often assumed that the magnetic moments are localized on the $\mathrm{Cu}$ atoms, with a moment of $\sim 1 . \mu_{B}$ per $\mathrm{Cu}$ and a Heisenberg coupling $\left(J S_{i} \cdot S_{j}\right)$ between nearest-neightor spins. Such a model can be tested in several ways. First of all, the intensity of magnetic Bragg diffraction peaks is proportional to the square of the stagsered magnetization $M_{0}$, which in turn is equal to $g \mu_{B}\left\langle S_{x}\right\rangle$, where $\left\langle S_{x}\right\rangle$ is the average spin on a $\mathrm{Cu}$ site. Spin-wave theory ${ }^{2}$ for the $2 \mathrm{D}, S=\frac{1}{3}$, square-lattice Heisenberg model at $T=0$ predicts that $\left\langle S_{z}\right\rangle=0.30$, so that, taking $g \approx 2.2, M_{A} \approx 0.67 \mu_{g}$. Experimentally it is observed that both the otaggered magnetization and the Neel temperature $T_{N}$ decrease rapidly with doping of the planes; however, as $T_{N}$ ap-

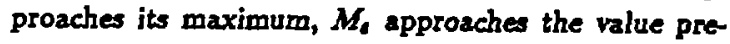

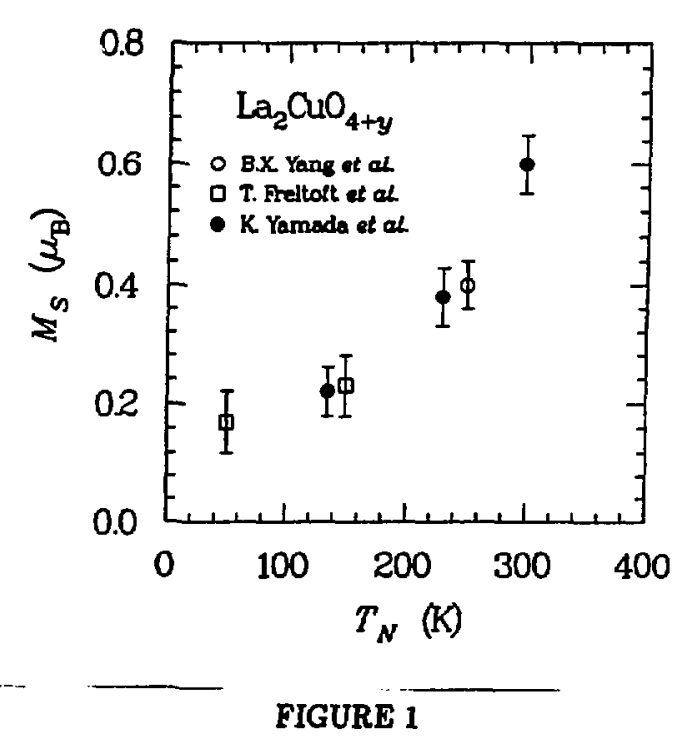

Staggared magnetiation vs. Néel temperature obtained by neutron diffaction measurements. Data is from Ref. 2.

dicted asouming full local moments, as shown in Fig. 1 for $\mathrm{La}_{2} \mathrm{CuO}_{4+y}$ (Ref. 2). Similar behavior has been observed in $\mathrm{YBa}_{2} \mathrm{Cu}_{3} \mathrm{O}_{6+2}$ (Ref. 3 and 4).

The magnetic Bragg peak intensities are also proportional to the square of the magnetic form factor, which is the Fourier transform of the spin density in real space. The $Q$ dependence of the antiferromagnetic form factor measured in a single crystal of $\mathrm{La}_{2} \mathrm{CuO}_{4+y}$ is in reasonable agreement with the $\mathrm{Cu}^{2+}$ form factor. ${ }^{5}$ Since by symmetry the spin density must be zero at the centers of the in-plane axygen atoms, it is not clear to what 


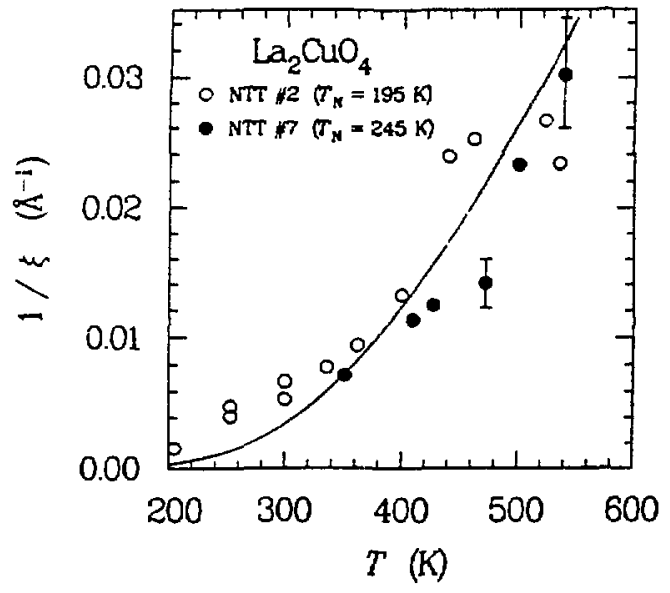

FIGURE 2

Temperature dependence of the inverse correlation length in $\mathrm{La}_{2} \mathrm{CuO}_{4}$. The data are from Ref. 12.

extent this result is or is not consistent with electronic structure calculations which indicate that the hole on each $\mathrm{Cu}$ atom contains a significant fraction of $\mathrm{O} 2 p$ character. To get a measure of the spin density on the in-plane oxygen atoms, one can attempt to measure the ferromagnetic form factor induced by an applied magnetic field. Such a study at $T>T_{N}$ in a $\mathrm{La}_{2} \mathrm{CuO}_{4+y}$ crystal indicated no significant contribution from the oxygens ${ }^{\circ}$; however, the applied field was much smaller than the effective exchange field between $\mathrm{Cu}$ atoms, so that it is difficult to properly compare the experimental results with theoretical calculations.

The superexchange interaction between neigliboring $\mathrm{Cu}$ atoms via the bridging oxygen atoms in the $\mathrm{CuO}_{2}$ planes is extremely strong. As a result, the spin-wave velocity $c=\omega / q$ is quite large, making it difficult to resolve the in-plane spin-wave branches at small $q$. Recently Aeppli et al. ${ }^{7}$ succeeded in resolving the $2 D$ spin waves using epithermal neutrons, obtaining a spin wave velocity $\hbar c=0.85 \pm 0.03 \mathrm{eV}-\mathrm{A}$. Taling into account quantum renormalization factors, that velocity corresponds to an exchange parameter of $135 \mathrm{meV}$, in good agreement with recent two-magnon Raman scattering measurements. ${ }^{8}$ Large 2D spin-wave velocities are also observed ${ }^{9,10}$ in antiferromagnetic $\mathrm{YBa}_{2} \mathrm{Cu}_{3} \mathrm{O}_{6+x}$.

The $3 \mathrm{D}$ magnetic ordering temperature in $\mathrm{La}_{2} \mathrm{CuO}_{4}$ is limited by the weal coupling between planes. Because of the extremely large in-plane coupling, the $2 D$ mag-

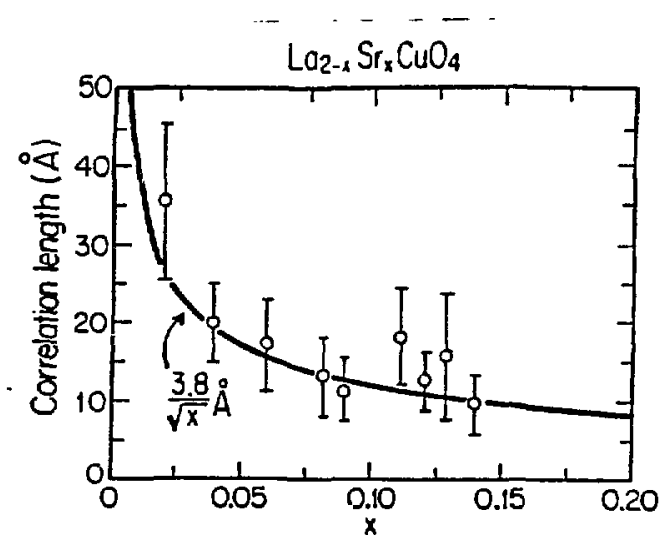

FIGURE 3

Antiferromagnetic correiation length vs. Sr concentration in $\mathrm{La}_{2-2} \mathrm{Sr}_{2} \mathrm{CuO}_{4}$. The solid line represents the average separation between the holes introduced by $\mathrm{Sr}$ doping. After Ref. 14.

netic correlation length $\xi$ remains large well above $T_{N}$. The correlation length can be determined by integrating over the inelastic magnetic scattering to obtain the Fourier transform of the instantaneous spin-spin correlation function. By taking advantage of the fact that the scattering from a $2 D$ system is independent of the momentum transfer perpendicular to the plane, one can perform the energy integration using a special scattering geometry..$^{11}$ The inverse correlation length as a function of temperature is shown in Fig. 2, as measured in two different crystals. 12 From a theoretical analysis of the 2D, $S=\frac{1}{2}$ Heisenberg model, Chakravarty et al. ${ }^{13}$ have shown that the inverse correlation length should equal $(2 / a) \exp \left(-2 \pi \rho_{j} / k_{B} T\right)$, where $a=3.8 \mathrm{~A}$ and $2 \pi \rho_{d}=0.576 \mathrm{hc} / \mathrm{a}$. The solid line in Fig. 2 corresponds to this formula with $\hbar c=0.85 \mathrm{eV}-\lambda$. The same type of temperature dependence is expected for a system of classical spins, but for $S=\frac{1}{2}$ the correlation length at a given temperature is greatly reduced due to quantum fluctuations.

The correlation length is reduced when holes are added to the planes by doping $\mathrm{La}_{2} \mathrm{CuO}_{4}$ with either $\mathrm{S}$ or $O$. The variation of $\xi$ vs. Sr content in $\mathrm{La}_{2} \mathrm{Sr}_{2} \mathrm{CuO}_{4}$ as determined by neutron scattering is shown in Fig. 3. A typical length scale for the hole-doping is the average hole separation, $a / \sqrt{x}$, and the correlation length is observed to closely follow this curve. The picture of antiferromagnetically coupled moments in which the correlation length decreases due to $02 p$ holes implic- 


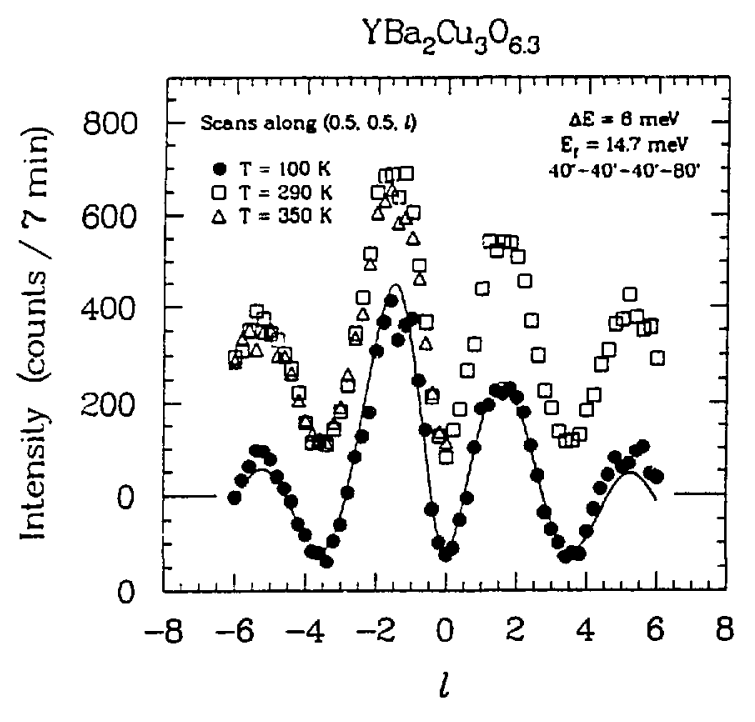

FIGURE 4

Scans along the 2D scattering rod at a constant energy transfer of $6 \mathrm{meV}$ measured in a single crystal of $\mathrm{YBa}_{2} \mathrm{Cu}_{3} \mathrm{O}_{6.3}$. From Ref. 9.

itly assumes that the $\mathrm{Cu}$ moments themselves are not changed by the doping. At small $x$, muon spin rotation experiments have shown that this is indeed the case. ${ }^{15}$ In metallic samples of $\mathrm{La}_{2-x} \mathrm{Sr}_{x} \mathrm{CuO}_{4}$ no direct measurement of the moments has been possible. To get a measure of the moments with neutron scattering, one would have to properly integrate the inelastic scattering up to the maximum excitation energies, which are presumably at least tenths of $\mathrm{eV}$, and such a measurement would be exceedingly difficult.

It would also be desirable to measure the magnetic correlation length in $\mathrm{YBa}_{3} \mathrm{Cu}_{3} \mathrm{O}_{6+3}$. Unfortunately, it is not possible to use the special scattering geometry which performs the energy integration because the inelastic scattering from the $\mathrm{CuO}_{2}$ layers is not independent of the momentum transfer perpendicular to the planes. The $\mathrm{Cu}$ atoms in the $\mathrm{CuO}_{2}$ layers do not form a Bravais lattice, and hence the spin waves are split into acoustic and optic modes, with each mode having a sinusoidal structure factor. The coupling between nearest-neighbor layers is sufficiently strong that one observes only the acoustic mode at energies up to at least $60 \mathrm{meV}, 9,10$ so that a scan at a low energy along the $2 \mathrm{D}$ scattering rod is strongly modulated by the acoustic mode structure factor, as illustrated in Fig. 4. The

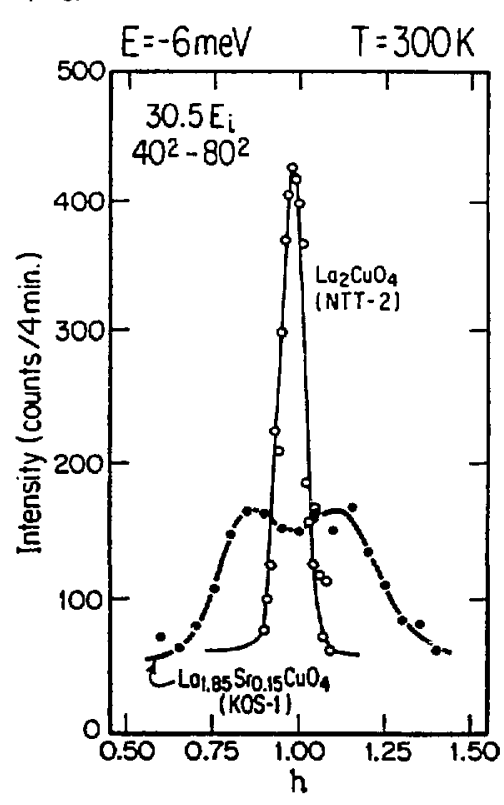

FIGURE 5

Direct comparison between the magnetic excitations of $\mathrm{La}_{2} \mathrm{CuO}_{4}$ and $\mathrm{La}_{1.85} \mathrm{Sr}_{0.15} \mathrm{CuO}_{4}$. From Ref. 16.

observation of the modulation at temperatures above $T_{N}$ indicates that the bilayers remain correlated in the absence of long-range order.

If magnetic excitations are directly connected with electron-pairing in superconducting samples, then one might expect to see some anomalous temperature dependence of the inelastic scattering at low temperatures. Measurements of inelastic scattering in metallic cuprate samples is difficult because, relative to antiferromagnetic samples, the intensity at a given energy is spread out over a much wider range in reciprocal space due to the short correlation length. Very large and good quality crystals are required for such work. Figure 5 compares scans across the 2D scattering rod at an energy transfer of $6 \mathrm{meV}$ for a superconducting crystal of $\mathrm{La}_{1.35} \mathrm{Sr}_{0.15} \mathrm{CuO}_{4}\left(T_{c}=33 \mathrm{~K}\right)$ and an antiferromagnetic $\mathrm{La}_{2} \mathrm{CuO}_{4}$ crystal. ${ }^{16}$ Although the peak-tobackground ratio is reduced in the doped crystal, the integrated intensity is essentially unchanged. An interesting feature is the development of incommensurate peaks, which so far have been observed only in superconducting crystals. ${ }^{17}$

Figure 6 shows the temperature dependence of the integrated intensity measured at energy transfers of 6 


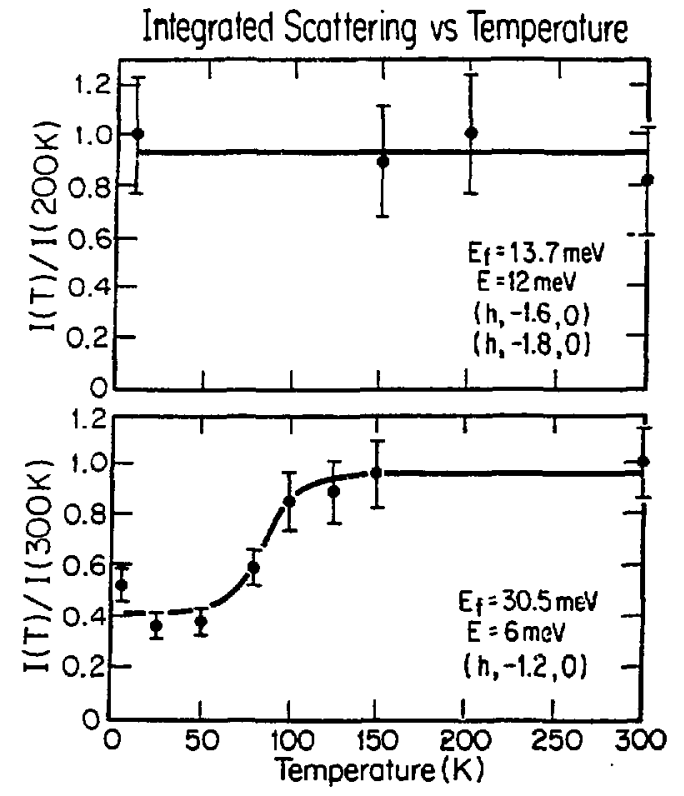

FIGURE 6

Temperature dependence of normalized integrated intensities at energy transfers of 6 and $12 \mathrm{meV}$ for $\mathrm{La}_{1.85} \mathrm{Sr}_{0.15} \mathrm{CuO}_{4}$. Solid lines are guides to the eye. From Ref. 16.

and $12 \mathrm{meV}$ in the $T_{c}=33 \mathrm{~K}$ crystal. ${ }^{16}$ In crystals with $T_{c} \sim 0$ the intensity of low energy scattering is essentially independent of temperature ${ }^{17}$ and the same behavior is observed at $12 \mathrm{meV}$ in the superconducting crystal. In contrast, a sharp decrease in intensity is found at $6 \mathrm{meV}$ between 150 and $50 \mathrm{~K}$. Similar behavior was also observed at 3 and $9 \mathrm{meV}$. If the intensity actually went to zero at low temperature, it would suggest that a gap had opened up in the low-energy magnetic excitation spectrum. That the intensity decrease occurs at temperatures well above $T_{c}$ is quite surprising; however, recent spin-lattice relaxation rate data from $\mathrm{Cu}$ NQR measurements by Imai et al. ${ }^{18}$ indicate a decrease in low-frequency spin fluctuations occurring in a similar temperature range.

Experiments are underway in search of magnetic excitations in superconducting $\mathrm{YBa}_{2} \mathrm{Cu}_{3} \mathrm{O}_{6+x}$, and we speculate that they will eventually be observed. A recent study ${ }^{19}$ has shown that strong $2 D$ antiferromagnetic correlations are also present above $T_{N}$ in $\mathrm{Nd}_{2} \mathrm{CuO}_{4}$; it will be quite interesting to learn how these correlations evolve on doping the compound with Ce. Clearly, neutron scattering will continue to play an important role in investigations of magnetisin in the layered cuprates.

\section{ACKNOWLEDGEMENTS}

Much of the work discussed here has been carried 0 : at Brookhaven in collaboration with many scientists, including R. J. Birgeneau, Y. Endoh, P. Gehring, Y. Hidaka, B. Keimer, H. Kojima, M. Sato, T. R. Thurston, and $K$. Yamada. The major part of this work was supported by the U.S.-Japan Cooperative Neutron Scattering Program Research at Brookharen National Laboratory is supported by the Division of Materials Sciences, U.S. Department of Energy, under Contract No. DE-AC02-76CH00016.

\section{REFERENCES}

1. P. W. Anderson, Phya. Rev. 86, 694 (1952).

2. B. X. Yang et al., J. Phya, Soc. Jpn. 56, 2283 (1987); T. Freitoft et al., Phys. Rev. B 38, 826 (1987); K. Yamada et ai., Solid State Commun. 64, 753 (1987).

3. J. M. Tranquada et al., Phys. Rev. B 38, 2477 (1988).

4. J. Rossat-Mignod et al., J. Phys. Colloq. 49, C8-2119 (1988).

5. T. Freltoft et al., Phys. Rev. B 37, 137 (1988).

6. C. Stassis et al., Phys. Rev. B 38. 9291 (1988).

7. G. Aeppli et al., Phys. Rev. Lett. 62, 2052 (1989).

8. R. R. P. Singh, P. A. Fleury, K. B. Lyons, and P. E. Sulewaki, Phys. Rev. Lett. 62, 2736 (1989).

9. M. Sato et al., Phys. Rev. Lett. 61, 1317 (1988); J. M. Tranquada et al., Phys. Rev. B (in press).

10. C. Vettier et al., Physica Scripta (in press).

11. G. Shirane et al., Phys. Rev. Lett. 59, 1613 (1987).

12. Y. Endoh et al., Phys. Rev. B 37, 7443 (1988); K. Yamada et al., Phys. Rev. B (to be published).

13. S. Chakravarty, B. I. Halperin, and D. R. Nelson, Phys. Rev. B 39, 2344 (1989).

14. R. J. Birgeneau et al., Phys. Rev. B 38, 6614 (1988); 39, 2868 (1989).

15. Y. J. Uemura et al., Pbys. Rev. Lett. 59, 1045 (1987); J. I. Budnick et al., Europhys. Lett. 5, 651 (1988).

16. G. Shirane et al., Phys. Rev. Lett. 63, 330 (1989).

17. T. Thurston et al., Phys. Rev. B (to be published).

18. T. Imaj et al., Phys. Rev. B (to be published).

19. Y. Endoh et al., Phys. Rev. B (to be published). 


\section{DISCLAIMER}

This report was prepared as an account of work sponsored by an agency of the United States Government. Neither the United States Government nor any agency thereof, nor any of their employees, makes any warranty, express or implied, or assumes any legal liability or responsibility for the accuracy, completeness, or usefulness of any information, apparatus, product, or process disclosed, or represents that its use would not infringe privately owned rights. Reference herein to any specific commercial product, process, or service by trade name, trademark, manufacturer, or otherwise does not necessarily constitute or imply its endorsement, recommendation, or favoring by the United States Government or any agency thereof. The views and opinions of authors expressed herein do not necessarily state or reflect those of the United States Government or any agency thereof. 